\title{
Spin-Isospin Resonances and Neutron Skin of Nuclei
}

\author{
D. Vretenar \\ Physics Department, Faculty of Science, University of Zagreb, Croatia \\ N. Paar \\ Physik-Department der Technischen Universität \\ München, D-85748 Garching, Germany and \\ Institut für Kernphysik, Technische Universität Darmstadt \\ Schlossgartenstr. 9, 64289 Darmstadt, Germany \\ T. Nikšić \\ Physics Department, Faculty of Science, University of Zagreb, Croatia, and \\ Physik-Department der Technischen Universität München, D-85748 Garching, Germany \\ P. Ring \\ Physik-Department der Technischen Universität München, D-85748 Garching, Germany
}

(Dated: July 19, 2018)

\begin{abstract}
The Gamow-Teller resonances (GTR) and isobaric analog states (IAS) of a sequence of even-even Sn target nuclei are calculated by using the framework of the relativistic Hartree-Bogoliubov model plus proton-neutron quasiparticle random-phase approximation. The calculation reproduces the experimental data on ground-state properties, as well as the excitation energies of the isovector excitations. It is shown that the isotopic dependence of the energy spacings between the GTR and IAS provides direct information on the evolution of neutron skin-thickness along the Sn isotopic chain. A new method is suggested for determining the difference between the radii of the neutron and proton density distributions along an isotopic chain, based on measurement of the excitation energies of the GTR relative to the IAS.
\end{abstract}

PACS numbers: 21.10.Gv, 21.30.Fe, 21.60.Jz, 24.30.Cz 
The determination of neutron density distributions in nuclei provides not only basic nuclear structure information, but it also places important additional constraints on effective interactions used in nuclear models. Various experimental methods have been used, or suggested, for the determination of the neutron density and differences between radii of the neutron and proton distributions [1, 2, 3, 4], but no existing measurement of neutron densities or radii has an established accuracy of one percent [5].

Potentially, a very accurate and model independent experimental method for the determination of neutron densities is the elastic scattering of longitudinally polarized electrons on nuclei. The electron interacts with a nucleus by exchanging either a photon or a $Z^{0}$ boson. Parity violation arises from the interference of electromagnetic and weak neutral amplitudes. The parity-violating asymmetry parameter, defined as the difference between cross sections for the scattering of right- and left-handed longitudinally polarized electrons, provides direct information on the Fourier transform of the neutron density [ $\underline{6}$ ]. A recent extensive analysis of possible parity-violating measurements of neutron densities and their theoretical interpretation can be found in Refs. [5, 7, 8]. Neutron density measurements will be also crucial for the analysis of atomic parity-violating (APV) experiments [5, 9, 10, 11]. Measurements of parity non conservation effects in intermediate and heavy atomic systems might provide very stringent tests of the Standard Model of electroweak interactions. At the present level of APV experimental precision, the dominant inaccuracy in the interpretation of the data is associated with atomic theory uncertainties in the electron density at the nucleus. These uncertainties could be considerably reduced by measuring APV observables for different atoms along an isotopic chain. Experiments involving isotope ratios, however, will be very sensitive to changes in the neutron density distribution along the isotopic chain. For atomic PV experiments it would be very important to determine with high accuracy $(\approx 1 \%)$ the neutron spatial distribution for a sequence of isotopes [11].

Krasznahorkay et al. have used the excitation of the giant dipole resonance (GDR) 2] and the excitation of the spin-dipole resonance (SDR) 4] to extract the neutron-skin thickness of nuclei. In Ref. 4], in particular, it has been demonstrated that there is a predictable correlation between the SDR cross section and the difference between the rms radii of the neutron and proton density distributions. By normalizing the results in the case of ${ }^{120} \mathrm{Sn}$, data on neutron-skin thickness along the stable Sn isotopic chain were obtained, in good agreement with theoretical predictions. 
In this Letter we suggest a new method for determining the difference between the radii of the neutron and proton density distributions along an isotopic chain, based on measurement of the excitation energies of the Gamow-Teller resonances relative to the isobaric analog states.

Nucleons with spin up and spin down can oscillate either in phase (spin scalar $\mathrm{S}=0$ mode) or out of phase (spin vector $\mathrm{S}=1$ mode). The spin vector, or spin-flip excitations can be of isoscalar $(\mathrm{S}=1, \mathrm{~T}=0)$ or isovector $(\mathrm{S}=1, \mathrm{~T}=1)$ nature. These collective modes provide direct information on the spin and spin-isospin dependence of the effective nuclear interaction (for an extensive review see Ref. [12]). Especially interesting is the collective spinisospin oscillation with the excess neutrons coherently changing the direction of their spins and isospins without changing their orbital motion - the Gamow-Teller resonance (GTR) $J^{\pi}=1^{+}$. The simplest charge-exchange excitation mode, however, does not require the spin-flip (i.e. $\mathrm{S}=0$ ) and corresponds to the well known isobaric analog state (IAS) $J^{\pi}=0^{+}$. The spin-isospin characteristics of the GTR and the IAS are related through the Wigner supermultiplet scheme. The Wigner SU(4) symmetry implies the degeneracy of the GTR and IAS, and furthermore the resonances would completely exhaust the corresponding sum rules [13]. The Wigner SU(4) symmetry is, however, broken by the spin-orbit term of the effective nuclear potential. In Ref. [13] it was noted that the energy difference between the GTR and the IAS decreases with increasing asymmetry $(N-Z) / A$.

It is implicit, therefore, that the energy difference between the GTR and the IAS reflects the magnitude of the effective spin-orbit potential. In the framework of relativistic mean-field (RMF) theory the spin-orbit term of the effective single-nucleon potential displays a strong isospin dependence: the magnitude of the spin-orbit potential is considerably reduced in neutron-rich nuclei 14]. This results in a reduction of the energy splittings between spin-orbit partner states. In Ref. [15] the relativistic Hartree-Bogoliubov (RHB) model was applied in the description of ground-state properties of Ni and Sn isotopes. The NL3 parameter set [16] was used for the effective mean-field Lagrangian, and pairing correlations were described by the pairing part of the finite range Gogny interaction D1S 17]. Fully self-consistent RHB solutions were calculated for the $\mathrm{Ni}(28 \leq N \leq 50)$ and $\mathrm{Sn}(50 \leq N \leq 82)$ isotopes. The resulting binding energies, neutron separation energies, and proton and neutron rms radii were found in excellent agreement with available experimental data. With the increase of the number of neutrons, the theory predicts a strong reduction of the spin-orbit potential (up 
to $\approx 30 \%$ in the surface region). The location of the minimum of the spin-orbit potential is also shifted outwards, and this is reflected in the larger spatial extension of the scalar and vector densities, which become very diffuse on the surface. The neutron-skin increases correspondingly. The issue of isospin dependence of the spin-orbit potential is, however, not quite settled among the various theoretical approaches (relativistic and non-relativistic) [18], and it is at the center of active experimental investigations in unstable nuclei.

In the following we shall demonstrate that there is a direct connection between the increase of the neutron-skin thickness in neutron-rich nuclei, and the decrease of the energy difference between the GTR and the IAS. The calculation is performed in the framework of the fully self-consistent RHB plus proton-neutron relativistic QRPA model. The RHB model represents a relativistic extension of the Hartree-Fock-Bogoliubov framework, and it provides a unified description of particle-hole $(p h)$ and particle-particle $(p p)$ correlations, that is essential for a quantitative analysis of ground-state properties and multipole response of unstable, weakly-bound nuclei far from the line of $\beta$-stability. Another relativistic model, the relativistic random phase approximation (RRPA), has been recently employed in quantitative analyses of collective excitations in nuclei. In Ref. [19] we have formulated the relativistic quasiparticle random phase approximation (RQRPA) in the canonical single-nucleon basis of the relativistic Hartree-Bogoliubov (RHB) model. By definition, the canonical basis diagonalizes the density matrix and it is always localized. This particular representation of the RQRPA is very convenient because, in order to describe transitions to low-lying excited states in weakly-bound nuclei, the two-quasiparticle configuration space must include states with both nucleons in the discrete bound levels, states with one nucleon in a bound level and one nucleon in the continuum, and also states with both nucleons in the continuum. The relativistic QRPA of Ref. [19] is fully self-consistent. For the interaction in the particle-hole channel effective Lagrangians with nonlinear meson self-interactions are used, and pairing correlations are described by the pairing part of the finite range Gogny interaction. Both in the $p h$ and $p p$ channels, the same interactions are used in the RHB equations that determine the canonical quasiparticle basis, and in the matrix equations of the RQRPA. The RQRPA configuration space includes also the Dirac sea of negative energy states.

In the meson-exchange picture of nucleon-nucleon forces it is the $\pi$ - and $\rho$-meson exchange that generate the spin-isospin dependent interaction terms. Even though in the RMF description of the nuclear ground state the direct one-pion contribution vanishes at 
the Hartree level because of parity conservation, the pion nevertheless plays an important role for excitations that involve spin degrees of freedom. Since it has a relatively small mass, the pion mediates the effective nuclear interaction over large distances. In the present relativistic description of spin-flip and isospin-flip excitations we employ the $\pi$ - and $\rho$-meson exchange in the $p-h$ residual interaction of the proton-neutron RQRPA. The first relativistic RPA calculations of isobaric analog resonances and Gamow-Teller resonances have been performed only recently 20]. This analysis was, however, restricted to doubly closed-shell nuclei. A rather small configuration space was used and, furthermore, configurations that include empty states from the negative-energy Dirac sea were neglected. A more complete relativistic RPA calculation of GT resonances in doubly closed-shell nuclei was reported in Ref. [21]. The ground states of ${ }^{48} \mathrm{Ca},{ }^{90} \mathrm{Zr}$ and ${ }^{208} \mathrm{~Pb}$ were calculated in the relativistic mean-field (RMF) model with the NL3 effective interaction. The spin-isospin correlations in the RRPA calculation of the GT response functions were induced by the isovector mesons $\pi$ and $\rho$. In addition to the standard non-linear NL3 effective interaction with the vector rho-nucleon coupling, the effective Lagrangian included the pseudo-vector pion-nucleon interaction, but not the rho-nucleon tensor term. Although the pion does not contribute in the RMF Hartree calculation of the ground state, it has a pronounced effect on the spin-isospin excitations. However, because of the derivative type of the pion-nucleon coupling, it is also necessary to include a zero-range Landau-Migdal term that accounts for the contact part of the nucleon-nucleon interaction. The analysis of Ref. 21] has shown that the RRPA calculation with the NL3 effective interaction, the pseudo-vector pion-nucleon coupling ( $m_{\pi}=138$ $\mathrm{MeV}$ and $\left.f_{\pi}^{2} / 4 \pi=0.08\right)$, and the Landau-Migdal force with the strength parameter $g_{0}^{\prime}=0.6$, nicely reproduces the experimental excitation energies of the main components of the GT resonances in ${ }^{48} \mathrm{Ca},{ }^{90} \mathrm{Zr}$ and ${ }^{208} \mathrm{~Pb}$.

In the present analysis we extend the approach of Ref. [21] and employ the self-consistent RHB plus proton-neutron relativistic QRPA to calculate the GTR and IAS in the Sn isotopic chain. The RMF effective interaction is NL3, the pion-nucleon interaction Lagrangian reads

$$
\mathcal{L}_{\pi N}=-\frac{f_{\pi}}{m_{\pi}} \bar{\psi} \gamma_{5} \gamma_{\mu} \partial^{\mu} \vec{\pi} \vec{\tau} \psi,
$$

and we also include the Landau-Migdal zero-range force in the spin-isospin channel of the residual $p-h$ interaction:

$$
V(1,2)=g_{0}^{\prime}\left(\frac{f_{\pi}}{m_{\pi}}\right)^{2} \vec{\tau}_{1} \cdot \vec{\tau}_{2} \boldsymbol{\Sigma}_{1} \cdot \boldsymbol{\Sigma}_{2} \delta\left(\boldsymbol{r}_{1}-\boldsymbol{r}_{2}\right) .
$$


With $g_{0}^{\prime}=0.6$ our model calculation reproduce the results of Ref. [21] for the GTR in doubly closed-shell nuclei. For open-shell nuclei pairing correlations are described by the pairing part of the finite range Gogny interaction D1S. As it has been shown in the RHB+RQRPA analysis of Ref. [19], the consistent inclusion of the pairing interaction both in the static RHB and in the dynamical linear response, is essential in order to satisfy the energy weighted sum rules and for the decoupling of spurious modes. In the present calculation we neglect possible proton-neutron pairing correlations. Even though these correlations can contribute to the low-lying GT strength responsible for $\beta$-decay, their effect on the main component of the GTR can be safely neglected.

In Fig. 1 w we display the calculated differences between the excitation energies of the main component of the Gamow-Teller resonances and the respective isobaric analog states for the sequence of even-even Sn target nuclei with $A=112-124$. The result of fully self-consistent RHB plus proton-neutron RQRPA calculations are shown in comparison with experimental data obtained in a systematic study of the $\left({ }^{3} \mathrm{He}, \mathrm{t}\right)$ charge-exchange reaction over the entire range of stable Sn isotopes [22]. The calculated energy spacings are in very good agreement with the experimental values, and this result clearly demonstrates that the relativistic meanfield plus RPA framework provides a very natural description of nuclear spin and isospin excitations. For lighter Sn isotopes it appears that the calculated values differ somewhat from the experimental trend but, of course, the theoretical energy spacings might depend on the details of the effective interaction. In fact, the experimental data shown in Fig. 1 provide very valuable information that can be used in constraining the spin-isospin channel of the effective interaction. In order to illustrate the agreement between the data and model results, in the insert of Fig. 1 the calculated excitation energies of the isobaric analog states are compared with experiment [22]. Except for ${ }^{112} \mathrm{Sn}$, the agreement between data and the calculated values of $E_{\mathrm{IAR}}$ is better than $\approx 0.5 \mathrm{MeV}$.

In Fig. 2 the calculated and experimental energy spacings between the GTR and IAS are plotted as a function of the calculated differences between the rms radii of the neutron and proton density distributions of even-even Sn isotopes (upper panel). The calculated radii correspond to the RHB NL3+D1S self-consistent ground-state solutions, on which the proton-neutron RQRPA calculations are performed. In the lower panel the calculated differences between neutron and proton rms radii are compared with available experimental data 4]. In Fig. 2 we notice a remarkable uniform dependence of the energy spacings 
between the GTR and IAS on the size of the neutron-skin. In principle, therefore, the value of $r_{n}-r_{p}$ can be directly determined from the theoretical curve for a given value of $E_{\mathrm{GT}}-E_{\mathrm{IAS}}$. This method is, of course, not completely model independent, but it does not require additional assumptions. Since the neutron-skin thickness is determined in an indirect way from the measurement of the GTR and IAS excitation energies in a sequence of isotopes, in practical applications at least one point on the theoretical curve should be checked against independent data on $r_{n}-r_{p}$.

In conclusion, we have employed the self-consistent RHB plus proton-neutron relativistic QRPA to calculate the spin-isospin excitations in a sequence of Sn isotopes. By using the NL3 parameter set for the effective RMF Lagrangian, the pairing part of the Gogny interaction D1S for the $T=1$ pairing channel, the pseudo-vector pion-nucleon interaction and the Landau-Migdal zero-range force with $g_{0}^{\prime}=0.6$ in the spin-isospin channel of the residual $p-h$ interaction of the proton-neutron RQRPA, it has been possible to reproduce experimental data on the excitation energies of the Gamow-Teller resonances relative to the isobaric analog states. We have also shown that the isotopic dependence of the energy spacings between the GTR and IAS provides direct information on the evolution of neutron skin-thickness along the Sn isotopic chain. Very good results have been obtained in comparison with available data on $r_{n}-r_{p}$. This analysis suggests that the neutron-skin thickness can be determined from the measurement of the excitation energies of the GTR relative to IAS.

[1] C. J. Batty, E. Friedman, H. J. Gils, and H. Rebel, Adv. Nucl. Phys. 19, 1 (1989).

[2] A. Krasznahorkay et al., Phys. Rev. Lett. 66, 1287 (1991); Nucl. Phys. A 567, 521 (1994).

[3] T. Suzuki et al., Phys. Rev. Lett. 75, 3241 (1995).

[4] A. Krasznahorkay et al., Phys. Rev. Lett. 82, 3216 (1999).

[5] C. J. Horowitz, S. J. Pollock, P. A. Souder, and R. Michaels, Phys. Rev. C 63, 025501 (2001).

[6] T. W. Donnelly, J. Dubach, and Ingo Sick, Nucl. Phys. A 503, 589 (1989).

[7] C. J. Horowitz, Phys. Rev. C 57, 3430 (1998). 
[8] D. Vretenar, P. Finelli, A. Ventura, G.A. Lalazissis, and P. Ring, Phys. Rev. C 61, 064307 (2000).

[9] M. J. Ramsey-Musolf, Phys. Rev. C 60, 015501 (1999).

[10] S. J. Pollock and M. C. Welliver, Phys. Lett. 464B, 177 (1999).

[11] D. Vretenar, G.A. Lalazissis, and P. Ring, Phys. Rev. C 62, 045502 (2000).

[12] F. Osterfeld, Rev. Mod. Phys. 64, 491 (1992).

[13] Yu. V. Gaponov and Yu. S. Lyutostanskii, Sov. J. Nucl. Phys. 19, 33 (1974); Sov. J. Part. Nucl. 12, 528 (1981).

[14] G.A. Lalazissis, D. Vretenar, W. Pöschl, and P. Ring, Phys. Lett. B 418, 7 (1998).

[15] G.A. Lalazissis, D. Vretenar, and P. Ring, Phys. Rev. C 57, 2294 (1998).

[16] G.A. Lalazissis, J. König, and P. Ring, Phys. Rev. C 55, 540 (1997).

[17] J. F. Berger, M. Girod and D. Gogny, Nucl. Phys. A428, 32 (1984).

[18] R.C. Nayak and J.M. Pearson, Phys. Rev. C 58, 878 (1998).

[19] N. Paar, P. Ring, T. Nikšić and D. Vretenar, Phys. Rev. C 67, 034312 (2003).

[20] C. De Conti, A. P. Galeão and F. Krmpotić, Phys. Lett. B 444, 14 (1998).

[21] Z-Y. Ma, B-Q. Chen, N. Van Giai, and T. Suzuki, arXiv:nucl-th/0308021.

[22] K. Pham et al., Phys. Rev. C 51, 526 (1995). 
FIG. 1: RHB plus proton-neutron RQRPA results for the energy spacings between the excitation energies of the main component of the Gamow-Teller resonances and the respective isobaric analog states for the sequence of even-even ${ }^{112-124} \mathrm{Sn}$ target nuclei. The experimental data are from Ref. [22]. In the insert the calculated excitation energies of the isobaric analog states are compared with data.

FIG. 2: The proton-neutron RQRPA and experimental 22] differences between the excitation energies of the GTR and IAS as a function of the calculated differences between the rms radii of the neutron and proton density distributions of even-even Sn isotopes. (upper panel) In the lower panel the calculated differences $r_{n}-r_{p}$ are compared with experimental data [4]. 